\title{
Antimethicillin-resistant Staphylococcus aureus and Biological Activities of Metabolites from Digitaria Sanguinalis L.
}

\author{
TAGHREED IBRAHIM ${ }^{1 *}$, A. A. EL-HELA², G. T. M. DAWOUD³ AND MONERA ZHRAN ${ }^{4}$
}

Department of Pharmacognosy, College of Pharmacy, King Saud University, Riyadh 11451, Saudi Arabia, ${ }^{1}$ Department of Pharmacognosy, Faculty of Pharmacy, Cairo University, Cairo 11562, '2Department of Pharmacognosy, Faculty of Pharmacy, Al-Azhar University, Cairo 11371, ${ }^{3}$ Department of Phytochemistry, Medicinal Plant Center, NODCAR, ${ }^{4}$ Department of Botany, Faculty of Science (Girls), Al-Azhar University, Cairo, Egypt

Ibrahim et al.: Biological Activities of Digitaria Sanguinalis L.

\begin{abstract}
The present research was aimed to evaluate the antimicrobial, antimethicillin-resistant Staphylococcus aureus, antiviral and cytotoxic activities of active fractions of the alcohol extract and seven isolated compounds from the aerial parts of Digitaria sanguinalis. Bioassay-guided fractionation of the ethanol extract of the aerial parts of Digitaria sanguinalis L. (Poaceae) followed by several chromatographic purification steps resulted in the isolation of seven compounds. Structural elucidation of isolated compounds was achieved on the basis of spectroscopic analysis (ultraviolet, infrared, ${ }^{1} \mathrm{H}$ and ${ }^{13} \mathrm{C}$ nuclear magnetic resonance and mass spectra). Antimicrobial, antimethicillin-resistant Staphylococcus aureus, antiviral and cytotoxic activities were determined for the n-hexane, ethyl acetate, n-butanol fractions of the ethanol extract, in addition to the isolated compounds. Seven compounds were isolated, p-coumaric acid (1) tricin (2), p-hydroxybenzoic acid (3), stigmasterol (4), $\beta$-sitosterol-3-O- $\beta$-D-glucoside (5), tricin-7-O- $\beta$-D-glucopyranoside (6) and isoorientin (7). The results revealed that most of the isolated compounds exhibited marked antimicrobial, antiviral and cytotoxic activities, while compounds $1,2,5$ and 6 showed a significant activity against methicillinresistant Staphylococcus aureus. This study revealed that several fractions and isolated compounds from Digitaria sanguinalis appear to be potential new therapeutic agents, due to their significant antimicrobial, antimethicillin-resistant Staphylococcus aureus, antiviral and cytotoxic activities.
\end{abstract}

Key words: Cytotoxic, Digitaria sanguinalis, MRSA, p-coumaric acid, tricin

Poaceae (Gramineae) is one of the largest vascular plant families, containing between 650 and 785 genera and 10000 species. Poaceae is also one of the most ecologically and economically important plant families ${ }^{[1]}$. Some Poaceae plants are used in folk medicine as antihypertensive, antidiabetic, antiinflammatory, anthelmintic, astringent, antiulcer, diuretic and antioxidant agents ${ }^{[2]}$. This family is of great economic and medicinal importance as it includes all cereals, bamboos and sugar cane. Steroids, terpenoids, volatile oils, saponins, flavonoids, phenolic acids, fatty acids, hydroxamic acids and alkaloids are the main classes of phytoconstituents in Poaceae ${ }^{[3]}$. Digitaria belongs to Poaceae family and is found in Egypt as three species under the local name Dafraa ${ }^{[4]}$.

One of the main causes of hospital-related mortality and morbidity is pathogenic bacterial infection ${ }^{[5]}$. The pathogen Staphylococcus aureus often colonizes the skin

*Address for correspondence E-mail: tshehata@ksu.edu.sa

July-August 2019 surface, the upper respiratory tract, and the nasal tract ${ }^{[6]}$. $S$. aureus accounts for high mortality rates in patients with pneumonia, endocarditis, sepsis and urinary tract infections ${ }^{[7]}$. Methicillin has been used to treat S. aureus infections since the 1950 s. Tragically, S. aureus acquired resistance towards methicillin after several years $^{[8]}$. Methicillin is a $\beta$-lactam antimicrobial that alters the penicillin-binding proteins required for blending of the peptidoglycans of $S$. aureus ${ }^{[9]}$. The occurrence of the MecA gene that encodes for PBP2a were utilized as benchmarks to identify the proximity of methicillin resistant $S$. aureus (MRSA) ${ }^{[10]}$. The

This is an open access article distributed under the terms of the Creative Commons Attribution-NonCommercial-ShareAlike 3.0 License, which allows others to remix, tweak, and build upon the work non-commercially, as long as the author is credited and the new creations are licensed under the identical terms

Accepted 28 May 2019

Revised 22 February 2019

Received 20 November 2018

Indian J Pharm Sci 2019;81(4):651-660 
development of MRSA infections is serious, as MRSA renders medicines ineffective and contributes to increased morbidity, mortality and healthcare costs ${ }^{[11]}$. Moreover, MRSA has high resistance rates against tetracycline, clindamycin, cotrimoxazole, rifampicin, macrolides and fluoroquinolones ${ }^{[12]}$. Several studies have identified alternative medications to respond MRSA, particularly by using natural products. Plants offer an assorted supply of biologically active components as potential healing compounds, including antimicrobials.

Digitaria sanguinalis L., known as hairy crabgrass, is a perennial, densely tufted grass with rhizomiferous main stems that is native to tropical Asia, the Middle East and parts of Africa. D. sanguinalis has been introduced to many other parts of the world for cultivation and has become naturalized in other locations, including Australia $^{[13]}$. Some Digitaria species were proven to possess antimicrobial and antioxidant activities ${ }^{[14]}$. The methanol leaf extract of Digitaria sanguinalis was proven to have moderate antiMRSA activity ${ }^{[15]}$. Veratric acid, maltol, and (-)-loliolide were isolated from Digitaria sanguinalis roots and showed changes in soil microbial community structure ${ }^{[16]}$. It is worth noting that there are no reports available regarding the phytochemical and biological properties of the aerial parts of Digitaria sanguinalis L. Therefore, it is important to examine the phytochemical and biological properties of different extracts and isolated compounds of the aerial parts of Digitaria sanguinalis.

\section{MATERIALS AND METHODS}

The aerial parts of Digitaria sanguinalis L. were collected from the garden of the Faculty of Pharmacy, Al-Azhar University, Cairo, Egypt in June 2013. The plant material was authenticated in the Botany Department, Faculty of Science, Al-Azhar University, Cairo, Egypt. The aerial parts of the plant were air dried, powdered and preserved at room temperature for chemical and biological investigation. A specimen of the aerial parts of Digitaria sanguinalis was deposited in the Pharmacognosy Department, Faculty of Pharmacy, Al-Azhar University, Cairo, Egypt.

\section{General experimental procedures:}

Melting points ( $\mathrm{mp}$, uncorrected) were determined using the electrothermal capillary melting point apparatus. UV spectra were determined on a Pye-Unicam spp. 1750 spectrophotometer (Cambridge, UK). IR spectra were obtained using $\mathrm{KBr}$ on a Philips PU-9712 infrared spectrophotometer and a Shimadzu IR-435. Electrospray ionization mass spectrometry (ESIMS) was carried out on a Xevo TQD triple quadrupole (Waters Corporation, Milford, MA 01757, USA) mass spectrometer. EIMS was carried on a Scan EIMS-TIC (158VG-ZAB-HF, X-.64, 800.00) mass spectrometer (VG Analytical, Inc.). The ${ }^{1} \mathrm{H}$ and ${ }^{13} \mathrm{C}$ nuclear magnetic resonance (NMR) measurements were obtained with a Bruker Avance III (400) NMR spectrometer operating at $400 \mathrm{MHz}\left(\right.$ for ${ }^{1} \mathrm{H}$ ) and $100 \mathrm{MHz}$ (for ${ }^{13} \mathrm{C}$ ) in $\mathrm{CD}_{3} \mathrm{OD}$ or dimethyl sulfoxide (DMSO)- $d_{6}$ solution; chemical shifts were expressed in $\delta(\mathrm{ppm})$ with reference to tri methyl silane (TMS) and with coupling constant $(J)$ in Hertz. Silica gel (Si gel 60, Merck, Germany) and Sephadex LH-20 (Pharmacia, Biotech, Sweden) were used for column chromatography. Solid phase extraction (SPE) was carried out on SPE-C18 cartridges (Strata column, Phenomenex, USA). Thinlayer chromatography (TLC) was carried out on precoated silica gel 60 F254 (Merck, Germany) plates. Chromatograms were visualized under UV light $(254 \mathrm{~nm})$ after spraying with ammonia, aluminium chloride solutions, or $1 \%$ vanillin $/ \mathrm{H}_{2} \mathrm{SO}_{4}$, followed by heating at $100^{\circ}$ for $5 \mathrm{~min}$. Shift reagents ${ }^{[17]}$, sodium metal, boric acid, hydrochloric acid, sodium acetate reagents and other chemicals used were of analytical grade.

\section{Extraction and isolation from plant material:}

Air-dried, powdered aerial parts of Digitaria sanguinalis $(2 \mathrm{~kg})$ was subjected to exhaustive extraction at room temperature with $70 \%$ ethanol $(81 \times 5,24 \mathrm{~h}$ each $)$. The combined ethanol extract was concentrated under vacuum at $40^{\circ}$ to dry. The concentrated ethanol extract $(140 \mathrm{~g})$ was then suspended in distilled water $(500 \mathrm{ml})$ and partitioned successively with $n$-hexane $(7 \times 500 \mathrm{ml}$, $30 \mathrm{~min}$ each), ethyl acetate $(5 \times 500 \mathrm{ml}, 30 \mathrm{~min}$ each) and $n$-butanol $(7 \times 500 \mathrm{ml}, 30 \mathrm{~min}$ each) to give $18.8 \mathrm{~g}$, $13.2 \mathrm{~g}$ and $16.5 \mathrm{~g}$, respectively. Aliquots of $n$-hexane fraction $(12 \mathrm{~g})$ were subjected to a silica gel column $(5 \mathrm{~cm}, 75 \mathrm{~cm}, 500 \mathrm{~g})$ using gradient elution with $n$-hexane-ethyl acetate 100:0 v/v (2 1), 90:10 v/v (3 1), $80: 20 \mathrm{v} / \mathrm{v}(21), 70: 30 \mathrm{v} / \mathrm{v}(31), 60: 40 \mathrm{v} / \mathrm{v}(21)$ and 50: $50 \mathrm{v} / \mathrm{v}(2 \mathrm{l})$ with a flow rate of $3 \mathrm{ml} / \mathrm{min}$; fractions of $100 \mathrm{ml}$ were collected and monitored by TLC. Six fractions were obtained: A $(510 \mathrm{mg}), \mathrm{B}(730 \mathrm{mg})$, C (450 mg), D (850 mg), E (933 mg) and F (810 mg). Fraction B was re-chromatographed on a silica gel column $(50 \mathrm{~cm}, 1 \mathrm{~cm}, 50 \mathrm{~g})$ eluted with $n$-hexane:ethyl acetate $(90: 10) \mathrm{v} / \mathrm{v}$ to give compound 1 (45 mg). Fraction D was chromatographed on a Sephadex 
LH-20 column $(50 \mathrm{~cm}, 1 \mathrm{~cm}, 30 \mathrm{~g})$ and eluted with $\mathrm{CH}_{2} \mathrm{Cl}_{2}: \mathrm{MeOH}(50: 50 \mathrm{v} / \mathrm{v}, 500 \mathrm{ml})$ to give compound $2(20 \mathrm{mg})$ and subfraction D-1 (90 mg). Subfraction D-1 was further subjected to SPE (RP-C18) using 50:50; 60:40 MeOH:water $\mathrm{V} / \mathrm{v}(500 \mathrm{ml}$, each) to obtain compound $3(25 \mathrm{mg})$. Fraction $\mathrm{E}$ was subjected to a Sephadex LH-20 column $(50 \mathrm{~cm}, 1 \mathrm{~cm}, 30 \mathrm{~g})$ and eluted with $\mathrm{MeOH}(500 \mathrm{ml})$ to afford compound 4 $(50 \mathrm{mg})$. From ethyl acetate fraction $(8 \mathrm{~g})$, compounds $5(8 \mathrm{mg})$ and $6(30 \mathrm{mg})$ were isolated using SPE-C18 cartridges eluted with methanol and increasing polarity with distilled water. The $n$-butanol fraction $(10 \mathrm{~g})$ was subjected to VLC $(75 \mathrm{~cm}, 5 \mathrm{~cm}, 350 \mathrm{~g})$ eluted with $\mathrm{CH}_{2} \mathrm{Cl}_{2}: \mathrm{MeOH} 95: 5$ to $30: 70 \mathrm{v} / \mathrm{v}(500 \mathrm{ml}$, each) to give five fractions: $\mathrm{G}(1.5 \mathrm{~g}), \mathrm{H}(2 \mathrm{~g}), \mathrm{I}(1.7 \mathrm{~g}), \mathrm{J}$ (3 g) and $\mathrm{K}(2.2 \mathrm{~g})$. Fraction $\mathrm{G}$ was rechromatographed on a silica gel column eluted with $\mathrm{CH}_{2} \mathrm{Cl}_{2}: \mathrm{MeOH}(95: 5 \mathrm{v} / \mathrm{v}$; $300 \mathrm{ml})$ to give three subfractions, G-1 (300 mg), G-2 (250 mg) and G-3 (430 mg). Subfraction G-2 was purified on a Sephadex LH-20 column $(50 \mathrm{~cm}$, $1 \mathrm{~cm}, 30 \mathrm{~g})$ and eluted with $\mathrm{MeOH}(200 \mathrm{ml})$ to obtain compound 7 (20 mg).

\section{Acid hydrolysis of compound 6:}

Five milligrams of compound 6 was refluxed with $2 \mathrm{M} \mathrm{HCl}$ in $\mathrm{MeOH}(5 \mathrm{ml})$ for $6 \mathrm{~h}$ at $80^{\circ}$. The reaction mixture was evaporated and after dilution with $\mathrm{H}_{2} \mathrm{O}$ $(10 \mathrm{ml})$, the hydrolysate was extracted with $\mathrm{CHCl}_{3}(3 \times$ $20 \mathrm{ml}, 10 \mathrm{~min}$ ). The $\mathrm{CHCl}_{3}$ extract was then evaporated to afford the aglycone. The aqueous layer was neutralized with $2 \mathrm{~N} \mathrm{KOH}$ solution and concentrated to $1 \mathrm{ml}$ under reduced pressure $\left(50^{\circ}\right)$, which was then compared with standard sugars by TLC in a solvent system consisting of $\mathrm{CHCl}_{3}: \mathrm{MeOH}: \mathrm{H}_{2} \mathrm{O} \quad(30: 12: 4$ $\mathrm{v} / \mathrm{v} / \mathrm{v})$.

\section{Antimicrobial assay:}

Antimicrobial activity of the $n$-hexane, ethyl acetate and $n$-butanol fractions of Digitaria sanguinalis and the isolated compounds was investigated in vitro using the diffusion agar technique ${ }^{[18]}$. The following bacterial and fungal strains were used: Gram-positive bacteria, Staphylococcus aureus (RCMB 010028) and Bacillis subtilis (RCMB 010067), Gram-negative bacteria, Escherichia coli (RCMB 010052) and Pseudomonas aeruginosa (RCMB 010043); fungal strains, Aspergillus fumigates (RCMB 02568) and Candida albicans (RCMB 05031). Ampicillin, gentamycin and amphotericin B were used as standard reference antimicrobial drugs. The microbial species are environmental and clinically pathogenic microorganisms obtained from Regional Center for Mycology and Biotechnology antimicrobial unit (RCMB) at Al-Azhar University, Cairo, Egypt. The tested samples were prepared in a concentration $1 \mathrm{mg} / \mathrm{ml}$ for fractions and $250 \mu \mathrm{g} / \mathrm{ml}$ for isolated compounds dissolved in DMSO. The results are presented in Table 1.

\section{Determination of MRSA isolates and antibiotic susceptibility:}

For MRSA differentiation from other $S$. aureus isolates, Muller-Hinton agar medium (Oxoid Oxoid Ltd., UK) was used. The strains in a liquid medium of $0.5 \mathrm{McF}$ arland standard concentrations were grown in Muller-Hinton agar medium, and antibiotic discs with ampicillin $(10 \mu \mathrm{g})$, erythromycin $(15 \mu \mathrm{g})$, penicillin (10 U), ciprofloxacin $(5 \mu \mathrm{g})$, clindamycin $(2 \mu \mathrm{g})$, vancomycin $(30 \mu \mathrm{g})$, methicillin $(5 \mu \mathrm{g})$ and gentamycin $(10 \mu \mathrm{g})($ Mast Group Ltd, UK) were then placed on the medium and incubated for $24 \mathrm{~h}$ at $37^{\circ[19]}$. The diameter of the clear zone around the discs was measured following Clinical Laboratory Standard Institute ${ }^{[20]}$ standards. The results are presented in Table 1.

\section{Antiviral assay:}

Screening of antiviral activity of $n$-hexane, ethyl acetate and $n$-butanol fractions of alcohol extract was conducted with the aerial parts of Digitaria sanguinalis, and the isolated compounds were then evaluated using cytopathic effect inhibition assay ${ }^{[21]}$. The assay was performed using hepatitis A virus (HAV), oral herpes simplex virus (HSV-1) and genital herpes simplex virus (HSV-2). The results are presented in Table 2.

\section{Cytotoxicity assay:}

The cytotoxicity of $n$-hexane, ethyl acetate and $n$-butanol fractions from the alcohol extract of the aerial parts of Digitaria sanguinalis as well as the isolated compounds were tested against three human tumour cell lines, hepatocellular carcinoma cells (HepG-2), colon carcinoma cells (HCT-116) and breast carcinoma cells (MCF-7), in addition to normal fibroblast cells (L929). The cells were obtained from the American Type Culture Collection (Rockville, MD, USA). The cells were grown on Roswell Park Memorial Institute 1640 medium (Nissui Pharm. Co., Ltd., Tokyo, Japan) and supplemented with $10 \%$ inactivated foetal calf serum with $50 \mu \mathrm{g} / \mathrm{ml}$ gentamycin. The cells were maintained at $37^{\circ}$ in a humidified atmosphere with $5 \% \mathrm{CO}_{2}$ and were sub cultured 2 to 3 times a week. The cytotoxic activity was determined using cell viability 
assay method ${ }^{[22]}$. The experiments were performed in triplicate and percent cell viability was calculated as the mean absorbance of control cells/mean absorbance of treated cells. The concentration-response curves were prepared, and the $\mathrm{IC}_{50}$ values were calculated and presented in Table 3.

\section{Statistical analysis:}

All experiments were replicated three times. The results are presented as the means \pm standard deviation. The statistical comparisons of data were performed using an ANOVA and t-test using SPSS 18.0 (IBM Corporation, US). Differences were considered significant at the $\mathrm{p}<0.001$ level.

\section{RESULTS AND DISCUSSION}

Structure elucidation data of isolated compounds is as follows, $p$-coumaric acid (1) colourless needles, UV $\chi_{\text {max }}(\mathrm{MeOH}) \mathrm{nm}: 207,340$; IR $v_{\max }(\mathrm{KBr}) \mathrm{cm}^{-1}: 3420$ $(\mathrm{OH}), 3100-2700(\mathrm{OH}), 1675(\mathrm{CO}), 1620,1600,1520$ $(\mathrm{C}=\mathrm{C}) ;{ }^{1} \mathrm{H}$ NMR $\left(\mathrm{CD}_{3} \mathrm{O} D, 400 \mathrm{MHz}\right) \delta 7.50(1 \mathrm{H}, d$, $J=16.0 \mathrm{~Hz}, \mathrm{H}-\beta), 7.36(2 \mathrm{H}, d, J=8.4 \mathrm{~Hz}, \mathrm{H}-2, \mathrm{H}-6)$, $6.73(2 \mathrm{H}, d, J=8.4 \mathrm{~Hz}, \mathrm{H}-3, \mathrm{H}-5), 6.25(1 \mathrm{H}, d, J=15.8$ $\mathrm{Hz}, \mathrm{H}-\alpha) ;{ }^{13} \mathrm{C}$ NMR $(\mathrm{CD} 3 \mathrm{O} D, 100 \mathrm{MHz}) \delta 167.66$ (CO), 159.90 (C-4), 145.59 (C- $\beta$ ), 129.88 (C-2, 6), 125.73 (C-1), 115.47 (C-3, 5), 113.43 (C- $\alpha)$; EIMS $m / z$ $164[\mathrm{M}]^{+}, 242$ (100\%), 178 (43\%), 120 (12.2\%), 40 $(19 \%)$.

Tricin (2)- yellow needles, UV $\chi_{\text {max }}(\mathrm{MeOH}) \mathrm{nm}: 245$, 269, 349, (NaOMe) nm: 264, $275 \mathrm{sh}, 330,416,\left(\mathrm{AlCl}_{3}\right)$ nm: $258 \mathrm{sh}, 278,305,372 \mathrm{sh}, 396\left(\mathrm{AlCl}_{3} / \mathrm{HCl}\right) \mathrm{nm}: 259$ sh, 279, 305, 364, 388, (NaOAc) nm: 264, 275 sh, 322, 362, (NaOAc/boric acid) nm: 260, $304 \mathrm{sh}, 343$; IR $v_{\text {max }}$ $(\mathrm{KBr}) \mathrm{cm}^{-1}: 3340(\mathrm{OH}), 1655(\mathrm{CO}), 1614,1505(\mathrm{C}=\mathrm{C}$ aromatic); ${ }^{1} \mathrm{H} \mathrm{NMR}\left(\mathrm{CD}_{3} \mathrm{O} D, 400 \mathrm{MHz}\right) \delta: 7.38(2 \mathrm{H}, \mathrm{s}$, H-2', 6 ) 6.73 (1H, s, H-3), 6.54-6.56 (1H, d, $J=1.6 \mathrm{~Hz}$, H-8), 6.19-6.21 (1H, d, J=1.7 Hz, H-6), 3.88 (6H, s, 3', 5 -OMe), $12.94(1 \mathrm{H}, \mathrm{s}, 5-\mathrm{OH}) ;{ }^{13} \mathrm{C}$ NMR (DMSO- $d_{6}$, $100 \mathrm{MHz}$ ) $\delta 182.26$ (C-4), 164.19 (C-2), 164.58 (C-7), 161.79 (C-5), 157.81 (C-9), 148.62 (C-3', 5'), 140.24 (C-4 ), 120.86 (C-1 '), 104.71 (C-2`, 6'), 104.00 (C-3), 104.16 (C-10), 99.33 (C-6), 94.71 (C-8), 56.80 (11, 12$\mathrm{OMe}) ;$ EIMS m/z $330[\mathrm{M}]^{+}, 331[\mathrm{M}+\mathrm{H}]^{+}$.

p-Hydroxy benzoic acid (3)- white solid, IR (KBr) $v_{\text {max }} 3450,3000,2850,2650,2550,1660,1590,1510$, 1450; ${ }^{1} \mathrm{H}$ NMR $\left(400 \mathrm{MHz}, \mathrm{C} D_{3} \mathrm{O} D\right) \delta 7.84(1 \mathrm{H}, \mathrm{d}$, $J=8.7, \mathrm{H} 2-\mathrm{H} 8), 6.78(1 \mathrm{H}, \mathrm{d}, J=8.7, \mathrm{H} 3-\mathrm{H} 5) ;{ }^{13} \mathrm{C} \mathrm{NMR}$ (100 MHz, CD $\mathrm{O} D) \delta 170.14$ (-COOH), 127.7 (C-1),
116.8 (C-3, 5), 163.32 (C-4), 130.95 (C-2, 6); ESIMS $\mathrm{m} / \mathrm{z} 137$ [M-H]'.

Stigmasterol (4)- white crystalline needles, IR $v_{\max }$ $(\mathrm{KBr}) \mathrm{cm}^{-1}: 3380(\mathrm{OH}), 2942$ and 2870 (aliphatic $\mathrm{CH}$ ), $1643(\mathrm{C}=\mathrm{C}) ;{ }^{1} \mathrm{H} \mathrm{NMR}\left(\mathrm{CDCl}_{3}, 400 \mathrm{MHz}\right) \delta 5.28(1 \mathrm{H}$, d, $J=4.6 \mathrm{~Hz}, \mathrm{H}-6), 5.14$ (1H, dd, $J=15.1,8.6 \mathrm{~Hz}, \mathrm{H}-22)$, 4.95 (1H, dd, $J=15.1,8.6 \mathrm{~Hz}, \mathrm{H}-23), 3.48$ (1H, m, H-3), $0.95(3 \mathrm{H}, \mathrm{d}, J=7.4 \mathrm{~Hz}, \mathrm{Me}-21), 0.91$ (3H, s, Me-19), $0.80(3 \mathrm{H}, \mathrm{t}, J=6.4 \mathrm{~Hz}, \mathrm{Me}-29), 0.77(3 \mathrm{H}, \mathrm{d}, J=7.4 \mathrm{~Hz}$, Me-26), $0.75(3 \mathrm{H}, \mathrm{d}, J=7.4 \mathrm{~Hz}, \mathrm{Me}-27), 0.60(3 \mathrm{H}, \mathrm{s}$, $\mathrm{Me}-18) ;{ }^{13} \mathrm{C}$ NMR $\left(\mathrm{CDCl}_{3}, 100.0 \mathrm{MHz}\right) \delta 140.53(\mathrm{C}-$ 5), 138.52 (C-20), 129.58 (C-21), 121.72 (C-6), 71.94 (C-3), 56.78 (C-14), 56.22 (C-17), 50.25 (C-9), 46.13 (C-22), 42.35 (C-4), 42.31 (C-13), 40.62 (C-18), 39.83 (C-12), 37.56 (C-1), 36.53 (C-10), 32.95 (C-2), 31.83 (C-7), 31.81 (C-8), 30.62 (C-25), 28.95 (C-16), 25.49 (C-23), 24.43 (C-15), 21.70 (C-19), 21.39 (C11), 20.22 (C-26), 19.82 (C-27), 19.33 (C-19), 12.25 (C-29), 11.96 (C-24); EIMS $m / z$ 412 [M] .

$\beta$-Sitosterol 3-O- $\beta$-D-glucoside (5)- white amorphous powder, IR (KBr) $v_{\max } 3432,1634 \mathrm{~cm}^{-1} ;{ }^{1} \mathrm{H}$ NMR (DMSO- $\left.d_{6}, 400 \mathrm{MHz}\right) \delta 0.68$ (s, Me-18), 0.86 (d, $J=6.5$ $\mathrm{Hz}, \mathrm{Me}-27), 0.89$ (t, $J=7.0 \mathrm{~Hz}, \mathrm{Me}-29), 0.92$ (d, $J=6.5$ $\mathrm{Hz}, \mathrm{Me}-26), 0.94$ (d, $J=6.5 \mathrm{~Hz}, \mathrm{Me}-21$ ), 0.97 (s, Me19), 3.12-3.39 (m, H-2'-H-5'), 3.43 (m, H-6 'b), 3.46 (m, H-3), 3.62 (dd, $J=10.7,5.8 \mathrm{~Hz}, \mathrm{H}-6$ a), 4.25 (m, H-1 ), 5.34 (m, H-6); ${ }^{13} \mathrm{CNMR}$ (DMSO- $d_{6}, 100 \mathrm{MHz}$ ) $\delta$ aglycon: 141.22 (C-5), 120.23 (C-6), 75.65 (C-3), 56.37 (C-14), 55.47 (C-17), 49.69 (C-9), 45.24 (C-24), 41.67 (C-13), 39.37 (C-4), 37.26 (C-12), 36.73 (C-1), 36.04 (C-10), 35.25 (C-20), 33.09 (C-22), 31.28 (C-7), 31.06 (C-8), 29.21 (C-2), 28.31 (C-25), 27.44 (C-16), 25.38 (C-23), 23.62 (C-15), 22.46 (C-28), 20.13 (C11), 19.74 (C-26), 19.03 (C-19), 18.72 (C-27), 18.45 (C-21), 11.22 (C-29) and 11.05 (C-18) ppm. Sugar: 100.87 (C-1'), 76.46 (C-3'), 76.33 (C-5'), 73.22 (C2'), 70.17 (C-4'), 62.43 (C-6') ppm. ESI-MS m/z 577 $[\mathrm{M}+\mathrm{H}]^{+}$

Tricin-7-O- $\beta-D$-glucopyranoside (6)- yellow amorphous powder, UV $\chi_{\max }(\mathrm{MeOH}) \mathrm{nm}: 254,270$, 344, (NaOMe) nm: 261, $300 \mathrm{sh}, 430,\left(\mathrm{AlCl}_{3}\right) \mathrm{nm}: 270$, $297 \mathrm{sh}, 344,385,\left(\mathrm{AlCl}_{3} / \mathrm{HCl}\right) \mathrm{nm}:$ 272, $297 \mathrm{sh}, 335$, 344, (NaOAc) nm: 255, $266 \mathrm{sh}, 350,410$, (NaOAc/ boric acid) nm: 254, 338; IR $v_{\max }(\mathrm{KBr}) \mathrm{cm}^{-1}: 3420$ $(\mathrm{OH}), 1653(\mathrm{CO}), 1618,1499(\mathrm{C}=\mathrm{C}$ aromatic $) ;{ }^{1} \mathrm{H}$ NMR (DMSO- $\left.d_{6}, 400 \mathrm{MHz}\right)$ aglycon $\delta 12.95(1 \mathrm{H}, \mathrm{s}$, 5-OH), 7.38 (2H, s, H-2', 6 ) , 6.93-6.98 (1H, s, H-3), 6.54- $6.50(1 \mathrm{H}$, brs, H-8), $6.48(1 \mathrm{H}$, brs, H-6), 3.89 $\left(6 \mathrm{H}, \mathrm{s}, 3^{`}, 5^{`}-\mathrm{OMe}\right)$, sugar $\delta 5.26(1 \mathrm{H}, \mathrm{d}, J=7.1 \mathrm{~Hz}$, 
H-1“), 3.72 (1H, m, H-6“a), 3.42 (1H, m, H-6 `b), 3.32 (1H, t, $J=9.6 \mathrm{~Hz}, \mathrm{H}-3$ ' '), 3.29 (1H, m, H-5 '), 3.27 $\left(1 \mathrm{H}, \mathrm{t}, J=8.6 \mathrm{~Hz}, \mathrm{H}-2{ }^{\prime \prime}\right), 3.15\left(1 \mathrm{H}, \mathrm{d}, J=8.7 \mathrm{~Hz}, \mathrm{H}-2{ }^{\prime \prime}\right)$; ${ }^{13} \mathrm{C}$ NMR (DMSO- $d_{6}, 100 \mathrm{MHz}$ ) aglycon $\delta 182.49$ (C4), 164.65 (C-2), 163.46 (C-7), 161.55 (C-5), 157.35 (C-9), 148.75 (C-3`, 5'), 141.50 (C-4'), 121.0 (C-1'), 105.83 (C-10), 105.00 (C-2', 6'), 104.10 (C-3), 99.97 (C-6), 95.75 (C-8), 56.83 133(3`, 5'-OMe), sugar $\delta$ 98.5 (C-1“'), 77.82 (C-5“), 76.95 (C-3”'), 73.62 (C2"), 70.12 (C-4”), 61.11 (C-6"); ESIMS (positive mode) $m / z 493[\mathrm{M}+\mathrm{H}]^{+},[\mathrm{M}+\mathrm{Na}]^{+}, 331$ [aglycon $\left.+\mathrm{H}\right]^{+}$, 353 [aglycon+Na] ${ }^{+}$; ESIMS (negative mode) $\mathrm{m} / \mathrm{z} 491$ [M-H]-, 329 [aglycon-H]. TLC analysis of the aqueous portion of the hydrolysate of compound (6) revealed the presence of glucose as sugar moiety.

Isoorientin (7)- yellow amorphous powder, UV $\chi_{\max }$ $(\mathrm{MeOH}) \mathrm{nm}: 240 \mathrm{sh}, 255,270,348$, (NaOMe) nm: 265, 275, 338, 402, $\left(\mathrm{AlCl}_{3}\right) \mathrm{nm}:$ 277, $298 \mathrm{sh}, 331,425$, $\left(\mathrm{AlCl}_{3} / \mathrm{HCl}\right) \mathrm{nm}: 264 \mathrm{sh}, 272,290,362,384,(\mathrm{NaOAc})$ nm: 275, 322, 393, (NaOAc/boric acid) nm: 265, 373, 432; IR $v_{\text {max }}(\mathrm{KBr}) \mathrm{cm}^{-1}: 3400(\mathrm{OH}), 1658(\mathrm{CO}), 1585$, $1510\left(\mathrm{C}=\mathrm{C}\right.$ aromatic); ${ }^{1} \mathrm{H}$ NMR (DMSO- $d, 400 \mathrm{MHz}$ ) aglycon $\delta 13.54\left(1 \mathrm{H}\right.$, brs, 5-OH), $7.42\left(1 \mathrm{H}\right.$, brs, $\left.\mathrm{H}-2{ }^{\prime}\right)$, 7.40 (1H, brs, H-6 ), 6.91 (1H, d, $\left.J=8.5 \mathrm{~Hz}, \mathrm{H}-5^{`}\right), 6.65$ $(1 \mathrm{H}, \mathrm{s}, \mathrm{H}-3), 6.58(1 \mathrm{H}$, brs, H-8), sugar $\delta 4.55(1 \mathrm{H}, \mathrm{d}$, $\left.J=9.5 \mathrm{~Hz}, \mathrm{H}-1^{\prime \prime}\right), 4.07$ (1H, t, $\left.J=9.0 \mathrm{~Hz}, \mathrm{H}-1^{\prime}{ }^{\prime \prime}\right), 3.70$ (1H, d, J=11.4 Hz, H-6“a), 3.45 (1H, m, H-6”b), 3.25 $\left(1 \mathrm{H}, \mathrm{t}, J=8.9 \mathrm{~Hz}, \mathrm{H}-3{ }^{\prime \prime}\right), 3.22$ (1H, t, $\left.J=8.5 \mathrm{~Hz}, \mathrm{H}-4{ }^{\prime \prime}\right)$, $3.14\left(1 \mathrm{H}, \mathrm{m}, \mathrm{H}-5^{\prime \prime}\right),{ }^{13} \mathrm{C}$ NMR (DMSO- $d 6,100 \mathrm{MHz}$ ) aglycon $\delta 182.45$ (C-4), 163.2 (C-2, C-7), 161.13 (C5), 156.63 (C-9), 152.425 (C-4'), 143.28 (C-3'), 122.35 (C-1'), 120.35 (C-6'), 116.33 (C-5'), 114.62 (C-2'), 104.73 (C-10), 103.56 (C-3), 102.89 (C-6), 94.13 (C8), sugar $\delta 82.22$ (C-5 '), 80.32 (C-3“'), 73.46 (C-1 '), 71.66 (C-2”), 70.44 (C-4”), 60.67 (C-6“); ESIMS (positive mode) $\mathrm{m} / z$ $471[\mathrm{M}+\mathrm{Na}]^{+}$; ESIMS (negative mode) $m / z 447$ [M-H]', 285 [aglycon-H]'.

All the tested fractions showed antimicrobial activity against all the tested microorganisms and exhibited no MRSA activity (Table 1). Ethyl acetate fraction showed the highest activity against Gram-positive, Gramnegative and fungi, followed by $n$-hexane then $n$-butanol fractions. $p$-Coumaric acid, tricin, $\beta$-sitosterol-3$O$-glucoside and tricin-7-O-glucopyranoside have shown to possess antiMRSA activity. $p$-Coumaric acid exhibited the highest activity followed by tricin-7- $O$ glucopyranoside then $\beta$-sitosterol-3-O-glucoside and tricin.

Isoorientin produced no antimicrobial activity against all the tested microorganisms. $p$-Coumaric acid showed the highest activity against Gram-positive bacteria, followed by tricin-7-O-glucopyranoside then $p$-hydroxybenzoic acid and $\beta$-sitosterol-3- $O$-glucoside.

Tricin exhibited no activity against $B$. subtilis, but showed significant activity against $S$. aureus

TABLE 1: ANTIMICROBIAL AND ANTIMRSA ACTIVITY OF DIFFERENT FRACTIONS AND ISOLATED COMPOUNDS FROM DIGITARIA SANGUINALIS

\begin{tabular}{|c|c|c|c|c|c|c|c|c|c|c|c|}
\hline \multirow{3}{*}{ Organism } & \multicolumn{11}{|c|}{ Diameter of inhibition zone \pm SD $(\mathrm{mm})$} \\
\hline & \multicolumn{3}{|c|}{ Fractions } & \multicolumn{7}{|c|}{ Isolated compounds } & \multirow[b]{2}{*}{ Standard } \\
\hline & $n$ - hexane & $\begin{array}{l}\text { Ethyl } \\
\text { acetate }\end{array}$ & n-butanol & 1 & 2 & 3 & 4 & 5 & 6 & 7 & \\
\hline \multicolumn{11}{|c|}{ Gram-positive } & Ampicillin \\
\hline S. aureus & $15.4 \pm 0.25^{*}$ & $16.9 \pm 0.58^{*}$ & $11.8 \pm 0.27^{*}$ & $28.3 \pm 0.62^{*}$ & $\begin{array}{l}19.5 \pm \\
0.41^{*}\end{array}$ & $\begin{array}{l}16.2 \pm \\
0.32^{*}\end{array}$ & $\begin{array}{l}13.5 \pm \\
0.65^{*}\end{array}$ & $\begin{array}{l}18.3^{ \pm} \\
0.63^{*}\end{array}$ & $26.6 \pm 0.47^{*}$ & NA & $28.9 \pm 0.14$ \\
\hline B. subtilis & $17.9^{*} \pm 0.37$ & $20.9^{*} \pm 0.25$ & $12.66^{*} \pm 0.64$ & $22.6 \pm 0.35$ & NA & $\begin{array}{c}17.4^{*} \pm \\
0.42\end{array}$ & $\begin{array}{c}14.2^{*} \pm \\
0.27\end{array}$ & $\begin{array}{c}16.7^{*} \pm \\
0.28\end{array}$ & $22.5^{*} \pm 0.38$ & NA & $28.3 \pm 0.37$ \\
\hline \multicolumn{11}{|c|}{ Gram-negative } & Gentamycin \\
\hline $\begin{array}{l}P . \\
\text { aeruginosa }\end{array}$ & $12.3 \pm 0.38^{*}$ & $15.2 \pm 0.58^{*}$ & $11.1 \pm 0.37^{*}$ & $30.6 \pm 0.36^{*}$ & NA & $\begin{array}{l}15.6 \pm \\
0.64^{*}\end{array}$ & $\begin{array}{l}12.4 \pm \\
0.48^{*}\end{array}$ & $\begin{array}{l}17.2 \pm \\
0.35^{*}\end{array}$ & $16.4 \pm 0.54^{*}$ & NA & $20.3 \pm 0.37$ \\
\hline E. coli & $13.7 \pm 0.26^{*}$ & $17.1 \pm 0.25^{*}$ & $12.6 \pm 0.63^{*}$ & $32.7 \pm 0.28^{*}$ & $\begin{array}{l}15.6 \pm \\
0.57^{*}\end{array}$ & $\begin{array}{l}13.3 \pm \\
0.37^{*}\end{array}$ & $\begin{array}{l}13.1 \pm \\
0.26^{*}\end{array}$ & $\begin{array}{l}16.2 \pm \\
0.74^{*}\end{array}$ & $18.5 \pm 0.56^{*}$ & NA & $21.4 \pm 0.25$ \\
\hline Fungi & & & & & & & & & & \multicolumn{2}{|r|}{ Amphotericin- } \\
\hline $\begin{array}{l}\text { A. } \\
\text { fumigates }\end{array}$ & $14.7 \pm 0.54^{*}$ & $18.2 \pm 0.44^{*}$ & $12.6 \pm 0.36^{*}$ & $18.6 \pm 0.73^{*}$ & NA & $\begin{array}{l}18.6 \pm \\
0.47^{*}\end{array}$ & $\begin{array}{l}12.6 \pm \\
0.28^{*}\end{array}$ & $\begin{array}{l}17.4 \pm \\
0.43^{*}\end{array}$ & $12.3 \pm 0.46^{*}$ & NA & $22.9 \pm 0.44$ \\
\hline C. albicans & $13.8 \pm 0.40^{*}$ & $20.9 \pm 0.25^{*}$ & $12.3 \pm 0.24^{*}$ & $17.5 \pm 0.36^{*}$ & NA & $\begin{array}{l}16.4 \pm \\
0.31^{*}\end{array}$ & $\begin{array}{l}11.4 \pm \\
0.44^{*}\end{array}$ & $\begin{array}{l}15.4 \pm \\
0.48^{*}\end{array}$ & $13.8 \pm 0.27^{*}$ & NA & $21.4 \pm 0.25$ \\
\hline \multicolumn{12}{|c|}{ S. aureus (MRSA) } \\
\hline $\begin{array}{l}\text { S. aureus } \\
\text { (MRSA) }\end{array}$ & NA & NA & NA & $34.4 \pm 0.58^{*}$ & $\begin{array}{l}17.2 \pm \\
0.53^{*}\end{array}$ & NA & NA & $\begin{array}{l}20.3 \pm \\
0.45^{*}\end{array}$ & $25.5 \pm 0.49^{*}$ & NA & \\
\hline
\end{tabular}

The testing was done using agar diffusion method. *Significant at $p<0.001$ NA: no activity 
and E. coli. p-Coumaric acid showed highest activity against Gram-negative microorganisms, followed by $\beta$-sitosterol-3- $O$-glucoside then tricin7-O-glucopyranoside, $p$-hydroxybenzoic acid and stigmasterol. $p$-Coumaric acid exhibited highest antifungal activity followed by $p$-hydroxybenzoic acid, followed by $\beta$-sitosterol-3-O-glucoside, stigmasterol and tricin-7-O-glucopyranoside.

All the tested fractions showed antiviral activity against $\mathrm{HAV}$, and $n$-hexane showed the highest activity followed by ethyl acetate, and $n$-butanol. $n$-Hexane and ethyl acetate showed antiviral activity against HSV-1, while $n$-butanol was inactive. Only $n$-hexane showed antiviral activity against HSV-2 (Table 2). $p$-Coumaric acid and stigmasterol showed no antiviral activity against all the tested viruses, while tricin showed antiviral activity against all the tested viruses. Tricin and tricin-7-Oglucopyranoside showed high antiviral activity against HAV and HSV-1, and isoorientin was proven to have antiviral activity against HAV; $p$-hydroxybenzoic acid

\section{TABLE 2: ANTIVIRAL ACTIVITY OF DIGITARIA SANGUNALIS FRACTIONS AND ISOLATED COMPOUNDS}

\begin{tabular}{|c|c|c|c|}
\hline \multirow{2}{*}{ Sample } & \multicolumn{3}{|c|}{ Tested Viruses } \\
\hline & HAV & HSV-1 & HSV-2 \\
\hline$n$-hexane fraction & $+++v e$ & ++ ve & + ve \\
\hline Ethyl acetate fraction & ++ ve & $++v e$ & -ve \\
\hline$n$-butanol fraction & + ve & -ve & -ve \\
\hline$p$-coumaric acid & -ve & -ve & -ve \\
\hline Tricin & $++v e$ & ++ ve & $++v e$ \\
\hline p-hydroxybenzoic acid & -ve & + ve & + ve \\
\hline Stigmasterol & -ve & -ve & -ve \\
\hline$B$-sitosterol-3-O-glucoside & -ve & + ve & -ve \\
\hline Tricin-7-O-glucoside & $++v e$ & ++ ve & -ve \\
\hline Isoorientin & + ve & -ve & -ve \\
\hline
\end{tabular}

and $\beta$-sitosterol 3-O-glucoside showed activity against HSV-1. Only tricin and $p$-hydroxybenzoic acid have exhibited antiviral activity against HSV-2.

$n$-Hexane and ethyl acetate fractions exhibited significant cytotoxic activity against HepG-2 and MCF-7 cell lines but had no significant effect against the HCT-116 cell line (Table 3). The ethyl acetate fraction produced a higher cytotoxic effect $\left(\mathrm{IC}_{50}-10.2\right.$ and $1.42 \mu \mathrm{g}$ against HepG-2 and MCF-7, respectively) than the $n$-hexane fraction $\left(\mathrm{IC}_{50}-11.8\right.$ and $5.85 \mu \mathrm{g}$ against HepG-2 and MCF-7, respectively).

Tricin-7-O- $\beta$-D-glucopyranoside showed the highest cytotoxic activity against HepG-2 $\left(\mathrm{IC}_{50}-9.7 \mu \mathrm{g}\right)$, followed by $p$-hydroxy benzoic acid, $\beta$-sitosterol$3-O-\beta$-D-glucoside, tricin and $p$-coumaric acid ( $\mathrm{IC}_{50^{-}}-12.3,13.2,13.5$ and $15.6 \mu \mathrm{g}$, respectively). Compound $\beta$-sitosterol-3-O- $\beta$-D-glucoside exhibited the highest cytotoxic activity against $\mathrm{MCF}-7\left(\mathrm{IC}_{50}{ }^{-}\right.$ $3.8 \mu \mathrm{g}$ ), followed by compounds tricin-7-O- $\beta$-Dglucopyranoside, $p$-coumaric acid, $p$-hydroxy benzoic acid and tricin $\left(\mathrm{IC}_{50^{-}}\right.$4.8, 7.3, 8.6 and $14.6 \mu \mathrm{g}$, respectively). Stigmasterol and isoorientin showed no significant activity against any of the tested cell lines, and none of the tested fractions and isolated compounds exhibited significant cytotoxic activity against the HCT-116 cell line. None of the tested extracts and isolated compounds showed cytotoxic effect against normal fibroblast cells (L929), indicating their safety towards normal cells.

Seven compounds (1-7) were isolated for the first time from the $n$-hexane, ethyl acetate and $n$-butanol fractions of the alcohol extract of the aerial parts of D. sanguinalis (fig. 1). The isolated compounds were identified by comparing their spectral data with the published literature ${ }^{[23-38]}$. They were identified as

TABLE 3: CYTOTOXICITY OF DIFFERENT FRACTIONS AND ISOLATED COMPOUNDS FROM DIGITARIA SANGUINALIS AGAINST HEPG-2, HCT-116 AND MCF-7 CELL LINES

\begin{tabular}{|c|c|c|c|c|c|c|c|c|c|c|c|}
\hline \multirow{3}{*}{ Cell line } & \multicolumn{11}{|c|}{$\mathrm{IC}_{50} \pm \mathrm{SD}(\mu \mathrm{g})$} \\
\hline & \multicolumn{3}{|c|}{ Fractions } & \multicolumn{7}{|c|}{ Isolated compounds } & \multirow{2}{*}{$\begin{array}{c}\text { Standard } \\
\text { Vinblastine }\end{array}$} \\
\hline & $n$-hexane & $\begin{array}{l}\text { Ethyl } \\
\text { acetate }\end{array}$ & $\begin{array}{c}n- \\
\text { butanol }\end{array}$ & 1 & 2 & 3 & 4 & 5 & 6 & 7 & \\
\hline -еpG-2 & $11.8 \pm 0.53^{*}$ & $10.2 \pm 0.48^{*}$ & $69.5 \pm 1.20$ & $\begin{array}{l}15.6 \pm \\
0.42^{*}\end{array}$ & $\begin{array}{l}13.5 \pm \\
0.27^{*}\end{array}$ & $\begin{array}{c}12.3^{ \pm} \\
0.3^{*}\end{array}$ & $\begin{array}{c}77.8 \pm \\
1.36\end{array}$ & $13.2 \pm 0.39^{*}$ & $9.7 \pm 0.16^{*}$ & $65.7 \pm 1.10$ & $0.01 \pm 0.002$ \\
\hline HCT-116 & $38.8 \pm 0.64$ & $29.5 \pm 0.44$ & $52.0 \pm 1.26$ & $\begin{array}{c}22.7 \pm \\
0.40\end{array}$ & $\begin{array}{l}42.6 \pm \\
0.47\end{array}$ & $\begin{array}{c}25.2 \pm \\
0.43\end{array}$ & $\begin{array}{c}156.3 \pm \\
1.57\end{array}$ & $18.5 \pm 0.26$ & $24.7 \pm 0.24$ & $76.3 \pm 1.32$ & $0.01 \pm 0.001$ \\
\hline MCF-7 & $5.85 \pm 0.28^{*}$ & $1.42 \pm 0.26^{*}$ & $43.4 \pm 0.78$ & $\begin{array}{l}7.3 \pm \\
0.47^{*}\end{array}$ & $\begin{array}{l}14.6 \pm \\
0.34^{*}\end{array}$ & $\begin{array}{l}8.6 \pm \\
0.27^{*}\end{array}$ & $\begin{array}{c}95.2 \pm \\
0.87\end{array}$ & $3.8 \pm 0.12^{*}$ & $4.8 \pm 0.09^{*}$ & $52.8 \pm 0.88$ & $0.01 \pm 0.002$ \\
\hline L929 & $64.2 \pm 0.34$ & $54.8 \pm 0.14$ & $<80$ & $\begin{array}{l}46.3 \pm \\
0.47\end{array}$ & $\begin{array}{c}48.6 \pm \\
0.46\end{array}$ & $\begin{array}{c}45.5 \pm \\
0.62\end{array}$ & $<80$ & $63.2 \pm 0.73$ & $58.6 \pm 0.55$ & $<80$ & ND \\
\hline
\end{tabular}

Cytotoxicity of different fractions and isolated compounds from Digitaria sanguinalis was evaluated using vinblastine as standard reference against HEPG-2, HCT-116 and MCF-7 cell lines. *Significant at $p<0.001$ ND: not determined 
<smiles>O=C(O)/C=C/c1ccc(O)cc1</smiles>

A<smiles></smiles>
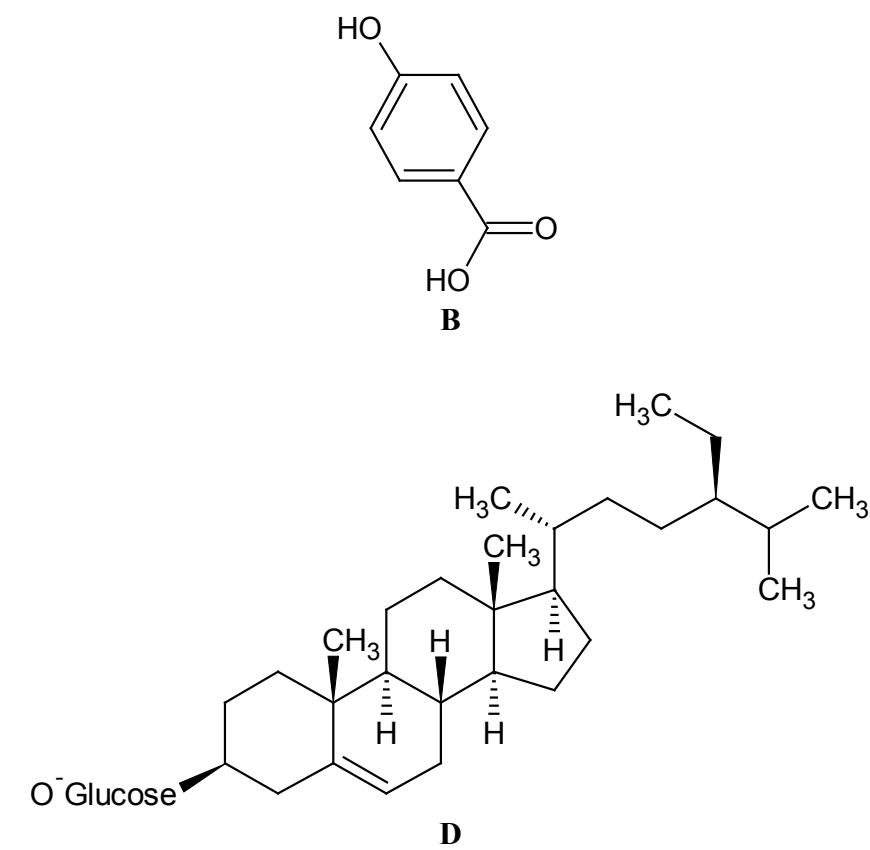

Fig. 1: Structure of the isolated compounds<smiles>[R20]c1cc2oc(-c3cc([R])c(O)c([R])c3)cc(=O)c2c(O)c1[R]</smiles>

(A) Compound (1); (B) compound (3); (C) compound (4); (D) compound (5); (E) compound (2): R1- H, R2- H, R3- OCH, R4$\mathrm{OCH}_{3}$; compound (6): R1- H, R2- glucose, R3- $\mathrm{OCH}_{3}$, R4- $\mathrm{OCH}_{3}$; compound (7): R1- glucose, R2- H, R3- OH, R4- H

p-coumaric acid (1), tricin (2), p-hydroxybenzoic acid (3), stigmasterol (4), which were isolated from the $n$-hexane fraction, $\beta$-sitosterol-3-O- $\beta$-D-glucoside (5), tricin-7-O- $\beta$-D-glucopyranoside (6), which were isolated from the ethyl acetate fraction and isoorientin (7), which was isolated from the $n$-butanol fraction.

The $n$-hexane and ethyl acetate fractions exhibited significant antimicrobial activity against the tested microorganisms, which may be attributed to the presence of $p$-coumaric acid and $p$-hydroxybenzoic acid in the $n$-hexane fraction and to the presence of $\beta$-sitosterol-3-O- $\beta$-glucoside and tricin-7-O- $\beta$ D-glucopyranoside in the ethyl acetate fraction, as these compounds were previously proved to have antimicrobial activity ${ }^{[39,40]}$. Isoorientin showed no antimicrobial activity supporting previously published findings ${ }^{[41]} . \quad p$-Coumaric acid showed significant antiMRSA activity again following previously published data ${ }^{[42]}$. However, this study is the first to find antiMRSA activity in tricin, $\beta$-sitosterol-3-O- $\beta$ glucopyranose and tricin-7-O- $\beta$-D-glucopyranoside.

The antimicrobial inhibitory mechanisms of phenolic compounds found to be active in this study may be due to iron deprivation or hydrogen bounding with vital proteins such as microbial enzymes ${ }^{[43]}$. Lipophilic flavonoids may disrupt microbial membranes whereas terpenes may have the ability to disrupt microbial membrane and this may explain their antimicrobial properties $^{[44]}$.

A study that investigated the relationship between structure, activity and the mechanism of action of phenolic acids as antimicrobial and antiMRSA agents found that phenolic acids (benzoic and cinnamic acid derivatives) possess antimicrobial activity, highlighting the importance of the carboxylic group in the molecule structure (proton acceptor), in addition to presence of $\mathrm{OH}$ (proton donor) and either $\mathrm{OCH}_{3}$ group (proton acceptor) or $\mathrm{H}$ in position 5 of the benzene ring. The 
docking studies provided strong evidence that the molecular basis for antiMRSA activity is probably due to PBP2a inhibitors ${ }^{[42]}$.

The $n$-hexane and ethyl acetate fractions showed significant antiviral activity against HAV and HSV-1, which may be attributed to the presence of tricin and tricin 7-O- $\beta$-D-glucopyranoside in the $n$-hexane and ethyl acetate fractions, respectively. Tricin presented a significant antiviral activity against HAV, HSV-1 and HSV-2; this result agrees with the published data, which proved the antiviral activity of tricin against influenza virus $^{[45]}$. Tricin-7-O- $\beta$-D-glucopyranoside presented a significant antiviral activity against HAV and HSV-1; the present study is the first study to demonstrate the antiviral activity of tricin-7-O- $\beta$-D-glucopyranoside, as previous study found no antiviral activity against respiratory syncytial virus $(\mathrm{RSV})^{[46]}$. This is also the first study to examine the antiviral properties of stigmasterol; however, it showed no antiviral activity. In addition, $p$-coumaric acid showed no antiviral activity, which agrees with previously published findings on the antiviral activity of $p$-coumaric acid ${ }^{[47]}$. $p$-Hydroxybenzoic acid was found to show weak antiviral activity against both HSV-1 and HSV-2, $\beta$-sitosterol-3-O-glucoside represented weak antiviral effect against HSV-1, and isoorientin was proven to have a weak effect against HAV; this study is the first antiviral study of $p$-hydroxybenzoic acid, $\beta$-sitosterol3-O-glucoside and isoorientin.

The $n$-hexane and ethyl acetate fractions exhibited significant cytotoxic activity against HepG-2 and MCF-7 cell lines, which may be attributed to the presence of $p$-coumaric acid and tricin in the $n$-hexane fraction and the presence of $\beta$-sitosterol-3-O- $\beta$ glucoside and tricin-7-O- $\beta$-D-glucopyranoside in the ethyl acetate fraction. $p$-Coumaric acid exhibited significant cytotoxic activity against HepG-2 and MCF-7 cell lines, and this result agrees with previous published studies, which proved that $p$-coumaric acid exhibited cytotoxic effect on neuroblastoma and H9c2 cadiomyoblasts $^{[48]}$. Tricin and tricin-7-O-glucosid presented significant cytotoxic activity against HepG2 and MCF-7, extending previous studies findings on their potent cytotoxic activity in vitro ${ }^{[49]}$. $\beta$-sitosterol3-O-glucoside exhibited significant cytotoxic activity once again confirming published data as $\beta$-sitosterol3-O-glucoside is known to possess significant cytotoxic activity towards the Caco- 2 cell line ${ }^{[50]}$. Stigmasterol, $p$-hydroxybenzoic acid and isoorientin exhibited no significant cytotoxic effect; however, no previous studies have examined the cytotoxic effect of $p$-hydroxybenzoic acid and isoorientin, but a previous study on stigmasterol found that it had no significant cytotoxic effect towards KB, CasKi, HCT 116, MCF7 and A549 cell lines ${ }^{[51]}$.

In this study, tricin's mechanism of cytotoxic action was examined to find that it was docked onto the indomethacin-bound structure of cyclooxygenase COX-2. It is considered to be an important mechanism of colorectal cancer chemoprevention. Ligand docking suggested that tricin can occupy the active site of COX $-2^{[52]}$. In prior work, Ovesna et al. ${ }^{[53]}$ stated that $\beta$-sitosterol and its glycoside derivatives can affect different levels of tumour development, such as their inhibitory effects on creation, promotion and induction of cancerous cells, as well as the inhibition of cell invasion and metastasis.

This study isolated seven compounds (1-7) from D. sanguinalis for the first time. Compounds $1,2,5$ and 6 showed significant antiMRSA and cytotoxic activities, while compounds 2 and 6 showed significant antiviral effects. The tested fractions and isolated compounds of $D$. sanguinalis demonstrated potential to be future therapeutic agents.

\section{Conflicts of interest:}

The authors declare no conflict of interest.

\section{Acknowledgements:}

This research project was supported by a grant from the Research Centre of the Female Centre for Scientific and Medical Colleges of King Saud University. Authors acknowledge the support of Professor Abdo Marey, Professor of Plant Taxonomy, Botany Department, Faculty of Science, Al-Azhar University, Cairo, Egypt in authenticating the plant material.

\section{REFERENCES}

1. Stanley K. Evolutionary trends in the grasses (Poaceae): a review. Mich Bot 1999;38:3-12.

2. Rathod J, Pathak N, Patel R, Jivani N, Bhatt N. Phytopharmacological properties of Bambusa arundinacea as a potential medicinal tree: An overview. J Appl Pharm Sci 2011;01:27-31.

3. Mohamed A, Abdelkader A, Sherif M, Mahmoud H. Chemical constituents and biological activities of the aerial parts of Stipagrostis plumosa (L) Munro ex T. Anderson. J Pharm Sci Res 2016;8:801-12.

4. Tackholm V. Students flora of Egypt. 2nd ed. Giza, Egypt: Cairo University; 1974. p. 657. 
5. Rice LB. Unmet medical needs in antibacterial therapy. Biochem Pharmacol 2006;71(7):991-5.

6. Stapleton PD, Taylor PW. Methicillin resistance in Staphylococcus aureus: mechanisms and modulation. Sci Prog 2002;85(1):57-72.

7. Kollef MH, Morrow LE, Niederman MS, Leepe KV, Anzueto A, BenzScott L, et al. Clinical characteristics and treatment patterns among patients with ventilator-associated pneumonia. Chest 2006;129(5):1210-8.

8. Jevons MP. “Celbenin”-resistant Staphylococci. Br Med J 1961;1:124-5.

9. Shurland S, Zhan M, Bradham DD, Roghmann MC. Comparison of mortality risk associated with bacteremia due to methicillin-resistant and methicillin-susceptible Staphylococcus aureus. Infect Control Hosp Epidemiol 2007;28(3):273-9.

10. Rachman A, Mat AN, Hui P, Suhaili Z, Amin NS, Othman Z, et al. Genotypic and phenotypic characterization of methicillin resistance determinants and $\beta$-lactamase in Staphylococcus species. Malays J Microbiol 2017;(13):308-17.

11. Cosgrove SE, Qi Y, Kaye KS, Harbarth S, Karchmer AW, Carmeli Y. The impact of methicillin resistance in Staphylococcus aureus bacteremia on patient outcomes: mortality, length of stay, and hospital charges. Infect Control Hosp Epidemiol 2005;26(2):166-74.

12. Kaleem F, Usman J, Hassan A, Omair M, Khalid A, Uddin R. Sensitivity pattern of methicillin resistant Staphylococcus aureus isolated from patients admitted in a tertiary care hospital of Pakistan. Iran J Microbiol 2010;2(3):143-6.

13. Duke J, Wain K. Medicinal plants of the world; computer index with more than 85,000 entries. Vol. 3. Harlow, United Kingdom: Longman Group Ltd.; 1981.

14. Kalaiyarasu $\mathrm{T}$, Karthi N, Sharmila V, Manju V. In vitro assessment of antioxidant and antibacterial activity of green synthesized silver nanoparticles from Digitaria radicosa leaves. Asian J Pharm Clin Res 2016;9:297-302.

15. Sharifi-Rad M, Iriti M, Sharifi-Rad M, Gibbons S, Sharifi-Rad J. Anti-methicillin-resistant Staphylococcus aureus (MRSA) activity of Rubiaceae, Fabaceae and Poaceae plants: A search for new sources of useful alternative antibacterials against MRSA infections. Cell Mol Biol 2016;62:39-45.

16. Zhou B, Kong $\mathrm{CH}$, Li $\mathrm{YH}$, Wang $\mathrm{P}, \mathrm{Xu} \mathrm{XH}$. Crabgrass (Digitaria sanguinalis) Allelochemicals that interfere with crop growth and the soil microbial community. J Agric Food Chem 2013;61(22):5310-7.

17. Ducrey B, Wolfender J, Marston A, Hostettmann K. Analysis of flavonol glycosides of thirteen Epilobium species (Onagraceae) by LC-UV and thermospray LCMS. Phytochemistry 1995;38:129-37.

18. Bauer A, Kirby W, Sheriss J, Turck M. Antibiotic susceptibility testing by standardized single method. Am J Clin Pathol 1966;45:493-6.

19. Clinical and Laboratory Standards Institute. Methods for dilution antimicrobial susceptibility tests for bacteria that grow aerobically; Approved standard. Wayne, Pennsylvania, USA: CLSI; 2006. Available from: https://clsi.org/media/1632/ m07a10_sample.pdf.

20. Clinical and Laboratory Standards Institute. Performance standard antimicrobial susceptibility testing; seventeenth informational supplement. Wayne, Pennsylvania, USA: CLSI; 2007. Available from: https://clsi.org/media/1469/m100s27_ sample.pdf.
21. Hu J, Hsiung G. Evaluation of new antiviral agents I: In vitro prospectives. Antiviral Res 1989;11:217-32.

22. Gangadevi V, Muthumary J. Preliminary studies on cytotoxic effect of fungal Taxol on cancer cell lines. Afr J Biotechnol 2007;6:1382-6.

23. Mohammed M, Ehab A, El-Hela A. Phytochemical investigation and biological evaluation of Dichanthium annulatum (Forrsk). J Sci Ind Res 2015;4:131-7.

24. Ramadoss K, Chapala D, Puttagunta SB. Isolation, characterization and RP-HPLC estimation of $p$-coumaric acid from methanolic extract of Durva grass (Cynodon dactylon Linn). Int J Anal Chem 2015;2015:201386.

25. Swisłocka R, Kowczyk-Sadowy M, Kalinowska M, Lewandowski W. Spectroscopic (FT-IR, FT-Raman, ${ }^{1} \mathrm{H}$ and ${ }^{13} \mathrm{C} \mathrm{NMR}$ ) and theoretical studies of $p$-coumaric acid and alkali metal $p$-coumarates. Spectroscopy 2012;27:35-48.

26. Yang Z, Nakabayashi R, Okazaki Y, Mori T, Takamatsu $\mathrm{S}$, Kitanaka S, et al. Towards better annotation in plant metabolomics: isolation and structure elucidation of 36 specialized metabolites from Oryza sativa (rice) by using MS/ MS and NMR analyses. Metabolomics 2014;10:543-55.

27. Wen P, Han H, Wang R, Wang N, Yao X. C-glycosylfavones and aromatic glycosides from Campylotropis hirtella (Franch.) Schindl. Asian J Tradit Med 2007;2:149-53.

28. Nakajima Y, Yun YS, Kunugi A. Six new flavonolignans from Sasa veitchii (Carr.) Rehder. Tetrahedron 2003;59:8011-5.

29. Mi Li, Yunqiao Pu, Chang GY, Arthur JR. The occurrence of tricin and its derivatives in plants. Green Chem 2016;18:1439.

30. Venkata $C$, Indra P. Isolation of stigmasterol and $\beta$-sitosterol from the dichloromethane extract of Rubus suavissimus. Int Curr Pharm J 2012;1:239-42.

31. Tania $\mathrm{P}, \mathrm{Kar} \mathrm{H}$. Isolation and characterization of $\beta$-Sitosterol3-O- $\beta$ - D-glucoside from the extract of the flowers of Viola odourata. Br J Pharm Res 2017;16:1-8.

32. Ram CD, Meena R, Surya KK, Suresh A, Mohan BG. Phytochemical constituents of the bark of Vitex negundo L. J Nepal Chem Soc 2008;23:89.

33. Kamboj A, Saluja AK. Isolation of stigmasterol and $\beta$ sitosterol from petroleum ether extract of aerial parts of Ageratum conyzoides (Asteraceae). Int J Pharm Pharm Sci 2011;3:94-6.

34. Chaurasia N, Wichtl M. Sterols and steryl glycosides from Urtica dioica. J Nat Prod 1987;50:881-5.

35. Voutquenne L, Lavaud C, Massiot G, Sevenet T, Hadi HA. Cytotoxic polyisoprenes and glycosides of long-chain fatty alcohols from Dimocarpus fumatus. Phytochemistry 1999;50:63-69.

36. Jing Z, Ying W, Zhang XQ, Zhang QW, Wen-Cai YE. Chemical constituents from the leaves of Lophatherum gracile. Chin J Nat Med 2009;7:428-31.

37. Lin YL, Kuo YH, Shiao MS, Chen CC, Ou JC. Flavonoid glycosides from Terminalia catappa L. J Chin Chem Soc 2000;47:253-6.

38. Peng J, Fan G, Hong Z, Chai Y, Wu Y. Preparative separation of isovitexin and isoorientin from Patrinia villosa Juss by high-speed counter-current chromatography. J Chromatogr A 2005;1074:111-5.

39. Naga A, Nadeem D, Pardha M, Mahendran B, Bharathi S. Cumulative activity of the $p$-coumaric acid and syringaldehyde for antimicrobial activity of different microbial strains. Eur J Exp Biol 2014;4:40-43.

40. Hassanein HD, Elsayed WM, Abreu AC, Simões M, Abdelmohsen MM. Polyphenolic constituents and 
antimicrobial activity of Rhapis excels (Arecaceae, Coryphoideae). Res J Pharm Biol Chem Sci 2015;6:1714-22.

41. Njinga NS, Pateh U, Hassan H, Abdullahi S, Ache R. Isolation and antimicrobial activity of $\beta$-Sitosterol-3-O-Glucoside from Lannea Kerstingii Engl. \& K. Krause (Anacardiacea). Nitte Univ J Health Sci 2016;6:4-8.

42. Alves MJ, Ferreira ICFR, Froufe HJC, Abreu RMV, Martins A, Pintado M. Antimicrobial activity of phenolic compounds identified in wild mushrooms, SAR analysis and docking studies. J Appl Microbiol 2013;115:346-57.

43. ScalbertA. Antimicrobial properties of tannins. Phytochemistry 1991;30:3875-83.

44. Cowan MM. Plant products as antimicrobial agents. Clin Microbiol Rev 1999;12:564-82.

45. Yazawa K, Kurokawa M, Obuchi M, Li Y, Yamada $\mathrm{R}$, Sadanari $\mathrm{H}$, et al. Anti-influenza virus activity of tricin, 4',5,7-trihydroxy-3',5'-dimethoxyflavone. Antivir Chem Chemother 2011;22:1-11.

46. Wang Y, Chen M, Zhang J, Zhang XL, Huang XJ, Wu X, et al. Flavone C-glycosides from the leaves of Lophatherum gracile and their in vitro antiviral activity. Planta Med 2012;78:46-51.

47. Schnitzler P, Neuner A, Nolkemper S, Zundel C, Nowack $\mathrm{H}$, Sensch $\mathrm{KH}$, et al. Antiviral activity and mode of action of propolis extracts and selected compounds. Phytother Res 2010;24:20-8.

48. Sunitha C, Kottayath N, Dhanyakrishnan R, Kumar BP. Protective effect of $p$-coumaric acid against doxorubicin induced toxicity in $\mathrm{H} 9 \mathrm{c} 2$ cardiomyoblast cell lines. Toxicol Rep 2015;2:1213-21.

49. Han JM, Kwon HJ, Jung HJ. Tricin, 4',5,7-trihydroxy-3',5'dimethoxyflavone, exhibits potent antiangiogenic activity in vitro. Int J Oncol 2016;49:1497-504.

50. Maiyo F, Moodley R, Singh M. Phytochemistry, cytotoxicity and apoptosis studies of $\beta$-Sitosterol-3-O-glucoside and $\beta$-amyrin from Prunus africana. Afr J Tradit Complement Altern Med 2016;13:105-12.

51. Malek SN, Shin SK, Wahab NA, Yaacob H. Cytotoxic components of Pereskia bleo (Kunth) DC. (Cactaceae) leaves. Molecules 2009;14:1713-24.

52. Scotti L, Bezerra Mendonça Junior FJ, Magalhaes Moreira DR, da Silva MS, Pitta IR, Scotti MT. SAR, QSAR and Docking of Anticancer Flavonoids and Variants: A Review. Curr Top Med Chem 2012;12(24):2785-809.

53. Ovesna Z, Vachalkova A, Horvathova K. Taraxasterol and betasitosterol: new naturally compounds with chemoprotective/ chemopreventive effects. Neoplasma 2004;51:407-14. 